EUROPEAN ORGANIZATION FOR NUCLEAR RESEARCH

European Laboratory for Particle Physics

Large Hadron Collider Project

LHC Project Report 513

\title{
THERMAL CONTRACTION MEASUREMENTS OF THE SUPERCONDUCTING COIL OF THE MAIN MAGNETS FOR THE LARGE HADRON COLLIDER
}

\author{
P. Ferracin, E. Todesco, D. Tommasini and W. Scandale
}

\begin{abstract}
We analyse the problem of defining and measuring the thermal contraction in the superconducting coil of the Large Hadron Collider main magnets. Since an unloaded coil length cannot be precisely defined, one has to use methods based on the pre-stress loss from room temperature to cryogenic temperature. A very strong dependence of the thermal contraction coefficient on the conventions used for defining coil deformations is discussed. Moreover, one finds different values of the thermal contraction coefficient according to the pressure imposed at room temperature before the cooling down. A pressure-dependent thermal contraction coefficient can be therefore worked out.
\end{abstract}

LHC Division

Presented at the 2001 Cryogenic Engineering Conference and International Cryogenic Materials Conference CEC/ICMC 2001

16-20 July 2001, Madison, Wisconsin, USA

Administrative Secretariat

LHC Division

CERN

CH - 1211 Geneva 23

Switzerland

Geneva, 19 October 2001 


\title{
THERMAL CONTRACTION MEASUREMENTS OF THE SUPERCONDUCTING COIL OF THE MAIN MAGNETS FOR THE LARGE HADRON COLLIDER
}

\author{
P. Ferracin, E. Todesco, D. Tommasini, and W. Scandale \\ CERN (European Laboratory for Nuclear Research) \\ 1211 Geneva 23, Switzerland
}

\begin{abstract}
We analyse the problem of defining and measuring the thermal contraction in the superconducting coil of the Large Hadron Collider (LHC) main magnets. Since an unloaded coil length cannot be precisely defined, one has to use methods based on the prestress loss from room temperature to cryogenic temperature. A very strong dependence of the thermal contraction coefficient on the conventions used for defining coil deformations is discussed. Moreover, one finds different values of the thermal contraction coefficient according to the pressure imposed at room temperature before the cooling down. A pressure-dependent thermal contraction coefficient can be therefore worked out.
\end{abstract}

\section{INTRODUCTION}

The coil of a superconducting magnet for particle accelerators [1-2] is surrounded by a mechanical structure that confines the conductors in a fixed geometry and exerts on them a pre-compression (pre-stress) along the coil azimuthal direction. The value of pre-stress is chosen to avoid cable motion due to electro-magnetic forces arising during the ramp-up of the magnet to the nominal field.

In the main dipole for the LHC [3], the particle accelerator under construction at CERN (Geneva), the mechanical structure which confines the coil consists of the austenitic steel collars, the iron yoke and shrinking cylinder (see FIGURE 1). The nominal pre-stress exerted by the collar on the coil at the end of the magnet assembly before the cool-down is of about $70 \mathrm{MPa}$. During the cool-down, a relevant pre-stress loss occurs and the azimuthal compression at $1.9 \mathrm{~K}$, the operating temperature, decreases to about $30 \mathrm{MPa}$ [4]. This loss is mainly due to the difference between the thermal shrinkage of the collars and that of the insulated cables [5-6]. 


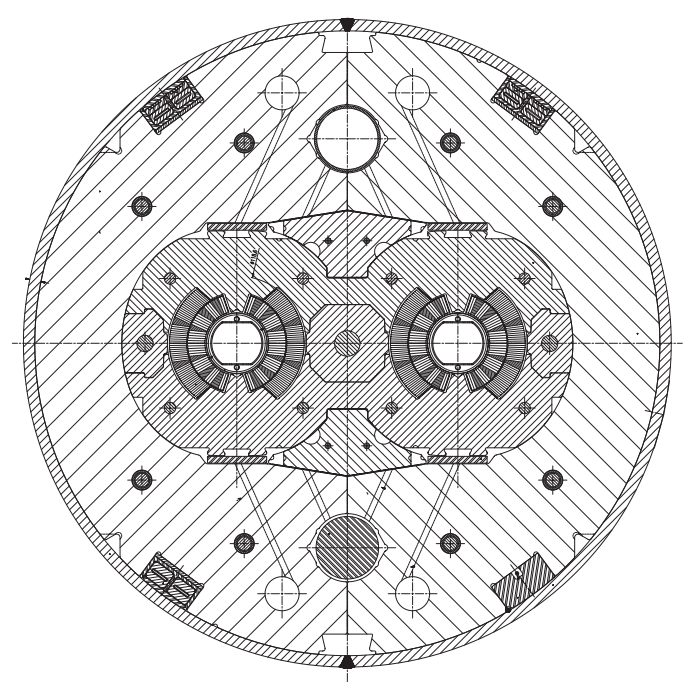

FIGURE 1. Cross-section of the LHC main dipole cold mass.

Therefore, for a correct modelling of the pre-stress losses and, consequently, of the mechanical behaviour of the magnet at $1.9 \mathrm{~K}$, a good knowledge of the thermal shrinkage of the coil is essential.

In this paper we present the measurements of the thermal contraction coefficient of a stack of conductors used in the outer layer coil of the LHC dipole [7]: the measurements have been performed taking into account the stress path followed by the coil during the magnet assembly.

\section{INTEGRATED THERMAL CONTRACTION COEFFICIENT}

\section{Definition and measurement methods}

The integrated thermal contraction coefficient can be defined as the relative difference of the unloaded height between two temperatures [8], i.e.

$$
\alpha=\frac{l_{w 0}-l_{c 0}}{l_{w 0}}
$$

Indeed, the unloaded height of a stack of insulated superconducting cable is ill-defined [9], equation (1) cannot be used for the evaluation of the thermal contraction coefficient of the stack in a direct manner. In fact, considering the stress-displacement curve presented in [7], at low pressure an increase of just $1 \mathrm{MPa}$ (for instance from $0 \mathrm{MPa}$ to $1 \mathrm{MPa}$ ) in the compression stress determines a strain of the same order of the thermal contraction of the stack [10-12], i.e. about $1 \%$.

We evaluated therefore the thermal shrinkage of the stack by measuring the stress losses in a mould (see for example [13]). In an infinitely rigid cavity of known integral thermal contraction coefficient $\alpha_{f}$, the mechanical deformation of the sample is equal to the difference in thermal contraction:

$$
\varepsilon_{w}-\varepsilon_{c}=\alpha_{s}-\alpha_{f}
$$


where $\alpha_{s}$ is the unknown thermal coefficient of the sample and $\mathcal{E}_{w}$ and $\mathcal{E}_{c}$ the deformations of the sample at ambient and cryogenic temperature. If the sample has a linear elastic behaviour, that is

$$
\varepsilon_{w}=\frac{\sigma_{w}}{E_{w s}} \quad \varepsilon_{c}=\frac{\sigma_{c}}{E_{w c}}
$$

one has

$$
\alpha_{s}=\frac{\sigma_{w}}{E_{w s}}-\frac{\sigma_{c}}{E_{c s}}+\alpha_{f}
$$

We generalise equation (4) to include also the non-linear behaviour of the stack presented in [7] and deformations of the mould. Let $E_{w f}$ and $E_{c f}$ be the elastic moduli of the cavity at warm and cold temperature respectively: then one has

$$
\alpha_{s}=\varepsilon_{w}\left(\sigma_{w}\right)+\frac{\sigma_{w}}{E_{w f}}-\varepsilon_{c}\left(\sigma_{c}\right)-\frac{\sigma_{c}}{E_{c f}}+\alpha_{f}
$$

To deduce the thermal contraction of the stack from the measurement of the stress loss in a fixed cavity by equation (5), one must know strains, and therefore also in this case one has to make assumptions on the unloaded coil height. Different assumptions on the unloaded stack height lead to very large differences in the strains values $\varepsilon_{w}\left(\sigma_{w}\right)$ and $\varepsilon_{c}\left(\sigma_{c}\right)$ of equation (5) and consequently on $\alpha_{s}$.

Moreover, strains significantly vary according to the $\sigma-\varepsilon$ relation assumed for the stacks: if we consider a simplified mechanical model of the stack with a linear behaviour, that is an elastic modulus independent on stress (see for instance $[9,13]$ ), we will obtain a much lower strain value, with respect to the realistic case of stress dependent elastic modulus, and thereafter a different $\alpha_{s}$. Finally, the hysteresis of the stress-strain curve at 77 $\mathrm{K}$ yields a further uncertainty to the problem. In fact, it is not clear how to evaluate $\varepsilon_{c}\left(\sigma_{c}\right)$ in equation (5), since it is not known if the stack reaches its final status at the end of the cool-down in loading or in unloading conditions.

One must conclude that the integral thermal contraction of a stack of film-insulated cables is not uniquely defined, as it depends on the scheme used to derive the strains from the displacements and on the stress path that the stack follows before the cool-down.

\section{EXPERIMENTAL SET-UP AND PROCEDURES}

We use a carbon steel mould: the stress is applied by a screw, placed at the top of the mould, and is measured by two capacitive gauges [16], placed at the top and the bottom of the stack. The sensitivity of the capacitive gauges is $1 \mathrm{MPa}$. In order to measure the stress loss from warm to cold temperature the device is covered by liquid nitrogen in a cryostat. The integral thermal contraction coefficient from $300 \mathrm{~K}$ to $77 \mathrm{~K}$ is computed through equation (5) by the measurement of the stress loss $\left(\sigma_{w}-\sigma_{c}\right)$ in the cavity.

Thermal contraction coefficient measurements have been calibrated by measuring stress losses with three different materials (aluminium $6082 \mathrm{~T} 6$, invar and copper) in our mould (see TABLE 1). 
TABLE 1. Integral thermal contraction coefficient $\alpha\left(10^{-3}\right)$ from $300 \mathrm{~K}$ to $77 \mathrm{~K}$ evaluated by the stress losses and comparison with reference values.

\begin{tabular}{lcc}
\hline & Measure & Reference \\
\hline Aluminum & $3.9 \pm 0.3$ & 3.92 \\
Invar & $0.4 \pm 0.1$ & 0.5 \\
Copper & $3.2 \pm 0.3$ & 3.04 \\
\hline
\end{tabular}

For the evaluation of the thermal contraction coefficient of the stack we use the following scheme: we performed five different cool-downs and we consider the stressdisplacement curves presented in [7] to determine the strain before and after the cool-down. The strains are then used to compute by equation (5) the thermal contraction.

At $300 \mathrm{~K}$ the stacks has been loaded to the peak stress and then unloaded to a value of stress $\sigma_{w}$ equal to the $60 \%$ of the peak stress. This procedure has been chosen taking into account the loading path followed by the coil during the LHC dipole assembly before the cool-down [15]. The strain $\mathcal{E}_{w s}$ has been computed as the difference between the stack height at 0.4 MPa on the loading curve (that we assumed as unloaded stack height [7]) and the height on the unloading curve at a stress of $\sigma_{w}$. At cryogenic temperature the strain $\varepsilon_{c s}$ can be evaluated in the same way. Since we do not know which status of compression is reached at the end of the cool-down, two estimates are computed: in the first case, by assuming that at $77 \mathrm{~K}$ the stack is in loading conditions, we describe the ideal case of a cool-down in absence of stress followed by the application of the stress up to $\sigma_{c}$. In the second case, by considering that the stack is in unloading conditions having reached a peak stress equal to $\sigma_{w}$, we describe another ideal case with a cool-down at constant stress $\sigma_{w}$, followed by a reduction of the stress from $\sigma_{w}$ to $\sigma_{c}$.

\section{RESULTS}

In FIGURE 2 we plot the thermal contraction coefficient of the conductor stack evaluated with the five cool-downs.

The error bar indicates the uncertainty associated to the hysteresis at $77 \mathrm{~K}$, that is the two ideal situations described above. The difference between the two cases is not negligible

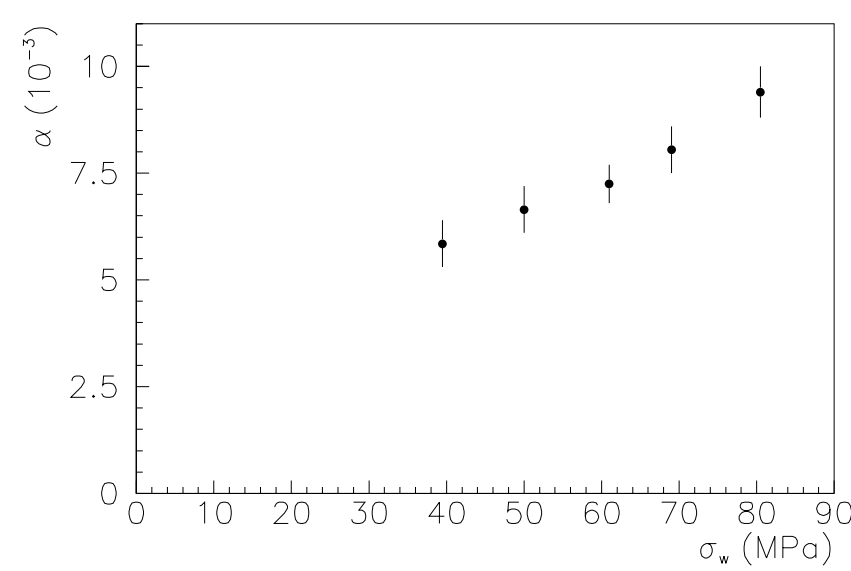

FIGURE 1. Integrated thermal contraction coefficient $\alpha$ versus stress $\sigma_{w}$ at $300 \mathrm{~K}$ for the conductor stack. 
and it is of about 0.001. Indeed, the dependence of $\alpha$ on the stress $\sigma_{w}$ is much larger than the error bars: one observes a variation from 0.006 for $\sigma_{w}=40 \mathrm{MPa}$ up to 0.009 for for $\sigma_{w}$ $=80 \mathrm{MPa}$. We conclude that the thermal contraction coefficient of the stack depends on the stress reached by the stack before the cool-down.

\section{CONCLUSION}

We have presented the results of measurements of the thermal contraction coefficient of a rectangular stack of insulated cables for the LHC dipole coil. We evaluated the thermal contraction coefficient measuring stress loss from five different stresses at $300 \mathrm{~K}$ obtaining results that range from 0.006 to 0.009 . Different assumptions on the trajectory in the $(\sigma, \varepsilon)$ plane followed during cool-down (either along loading or unloading curve) lead to additional variations of around 0.001 . We conclude that the thermal contraction coefficient significantly depends on the scheme used to derive it from measurements. This justifies the rather wide range of results that can be found in the literature.

\section{ACKNOWLEDGEMENTS}

We wish to acknowledge Shlomo Caspi for discussing with us about the thermal contraction coefficient of insulated superconducting cables. We would like to thank Arnaud Devred for several discussions on the mechanical properties of superconducting cables and on the problem of pre-stress losses in superconducting magnet.

\section{REFERENCES}

1. Mess, K. -H., Schmuser, P. and Wolff, S., Superconducting Accelerator Magnets, World Scientific, Singapore, 1996.

2. Wilson, M. N., Superconducting Magnets, Oxford, U.K.: Clarendon, 1986.

3. Artoos, K., et al., IEEE Trans. Appl. Superconduct. 10, pp.98-102 (1999).

4. Ferracin, P., et al., "Modelization of the thermo-mechanical structure of the LHC main dipole and influence on field quality", in Seventh European Accelerator Conference, edited by C. Petit-Jean Genaz, IoP, New York, 2000, pp. 330-332.

5. Swenson, C. A., Dixon, I. R. and Markiewicz, W. D., IEEE Trans. Appl. Superconduct. 7, pp. 408-411 (1997).

6. Chow, K. P. and Millos, G. A., IEEE Trans. Appl. Superconduct. 9, pp. 213-215 (1999).

7. Couturier, K., et al., "Elastic modulus measurements of the LHC dipole superconducting coil at $300 \mathrm{~K}$ and at $77 \mathrm{~K} "$, in this Conference.

8. Touloukian, Y. S., Kirby, R. K., Taylor, R. E. and Desai, P. D., "THERMAL EXPANSION. Metallic Elements and alloys", Thermophysical properties of matter 12, IFI/PLENUM, New York-Washington.

9. Meuser, R. B., Caspi, S. and Gilbert, W. S., IEEE Trans. Magn. 17, (1981).

10. Devred, A., et al., "About the mechanics of SSC dipole magnet prototypes", SSCL-Preprint-6, 1991.

11. Goodzeit, C., "Thermal contraction measurements", BNL-MDN-445-15, 1992.

12. Gladky, V. N., Kozub, S. S., Veshchikov, A. T. and Escher, U., Cryogenics 35, pp. 67-69 (1995).

13. Reytier, M., Devred, A., Durante, M., Gourdin, C. and Vedrine, P., "Characterisation of the thermomechanical behaviour of insulated cable stacks representative of accelerator magnet coils", DAPNIASTCM-00-23, 2000.

14. Siegel, N., Tommasini, D. and Vanenkov, I., "Design and use of capacitive force transducers for superconducting magnet models for the LHC", CERN-LHC-Project-Report-173, 1998.

15. Andreev, N., et al., "Mechanical behaviour of the short models of LHC main dipole magnets", $C E R N$ LHC-Project-Report-179, 1998. 